\title{
Neonatal Abstinence Syndrome Due to Opioid Abuse in Pregnancy
}

\author{
Banu MUTLU OZYURT'1, Ozge SURMELI ONAY', Ozlem ERSOY²
}

Mersin, Turkey

\begin{abstract}
Opioid abuse during pregnancy is increasing in women of childbearing age in Turkey. The frequency of clinical signs of withdrawal in infants who exposed to heroin in utero are varying between $16-90 \%$. Here, we present five newborn infants presenting with neonatal abstinence syndrome who were hospitalized in Neonatal Intensive Care Unit of Mersin Maternity and Children's Hospital. All of the five infants were symptomatic including irritability, tremors, high-pitched cry, excessive sucking and seizure. Hyperirritability was the predominant sign. Seizure was observed two of the five infants which clinically presented between $1^{\text {st }} 5^{\text {th }}$ day of life and was controlled with phenobarbital. We have experienced seizure due to withdrawal of opioid more than the past reports and we thought that interrupting breastfeeding may facilitate seizure. Breastfeeding may slow down the decrease of opioid level in blood and may reduce the symptoms. In conclusion, opioid abuse in pregnancy is a growing sociological problem in Turkey and a protocol for management is required for neonatologists.
\end{abstract}

Keywords: Opioid abuse, Neonatal abstinence syndrome, Seizure, Breastfeeding

Gynecol Obstet Reprod Med 2018;24(3):170-174

\section{Introduction}

Neonatal abstinence syndrome (NAS) is a group of problems that occur in a newborn who was exposed to addictive illegal or prescription drugs while in the mother's womb. Two major types of NAS are recognized: NAS due to prenatal or maternal use of substances that result in withdrawal symptoms in the newborn and postnatal NAS secondary to discontinuation of medications such as fentanyl or morphine used for pain therapy in the newborn. Some of the drugs and substances that cause withdrawal syndrome are codeine, heroin, methadone,

${ }_{1}^{1}$ Division of Neonatology Department of Pediatrics Mersin Maternity and Children's Hospital, Mersin

2 Department of Pediatrics Mersin Maternity and Children's Hospital, Mersin

Address of Correspondence: Ozge Surmeli Onay

Division of Neonatology, Department of

Pediatrics, Mersin Maternity and

Children's Health and Diseases

Hospital 33240 Mersin, Turkey.

e-mail: ozgeonay79@gmail.com

Submitted for Publication:

19. 02.2017

Accepted for Publication:

18. 12.2017

\begin{tabular}{|c|c|}
\hline & Access this article online \\
\hline $\begin{array}{c}\text { Quick Response Code: } \\
\text { Website: www.gorm.com.tr } \\
\text { info@gorm.com.tr }\end{array}$ \\
\cline { 2 - 2 }
\end{tabular}

How to cite this article: Mutlu Ozyurt B. Surmeli Onay O. Ersoy O. Neonatal Abstinence Syndrome Due to Opioid Abuse in Pregnancy. Gynecol Obstet Reprod Med 2018;24(3):170-174 meperidine, morphine, phenobarbital, alcohol, amphetamines, cocaine, clomipramine, imipramine, diazepam, hydroxyzine, and selective serotonin reuptake inhibitors (1).

Opioid abuse during pregnancy is increasing in women of childbearing age in Turkey. Intrauterine exposure to opioids may cause congenital anomalies and/or fetal growth restriction, increase the risk of preterm birth, produce signs of withdrawal or toxicity in the neonate, or impair normal neurodevelopment. The frequency of clinical signs of withdrawal in infants who exposed to heroin in utero are varying between $16-90 \%$ (2). These infants may require prolonged treatment and weeks of hospitalization. Symptoms and signs resulted in heroin withdrawal are as follows; irritability, hyperactivity, tremors, high-pitched cry, excessive sucking and seizure. In the literature, seizure has been reported in $2-11 \%$ while abnormal EEG results without overt convulsion activity have been reported in $>30 \%$ of newborn infants withdrawing from opioids (3).

Different scoring systems have been developed for assessing the severity of neonatal abstinence syndrome, such as those by Finnegan, Ostrea, Lipsitz, Rivers, and the Neonatal Intensive Care Unit Network Neurobehavioral Scale (NNNS) $(4,5,6)$. The most widely used system is the Finnegan scoring system, in both its original and modified forms. Here, we present five newborn infants presenting with NAS based on Finnegan scoring system. The demographic and clinical characteristics of the infants were summarized in table 1 . 
Table 1: The demographic and clinical characteristics of the infants who were born from heroin addicted mothers

\begin{tabular}{|c|c|c|c|c|c|}
\hline & Case 1 & Case 2 & Case 3 & Case 4 & Case 5 \\
\hline Substance use & Heroin & Heroin & Heroin & Heroin & Heroin \\
\hline Gestational age (week) & 35 & 38 & 39 & 32 & 39 \\
\hline Gender & Male & Female & Male & Female & Male \\
\hline Mode of delivery & $\mathrm{C} / \mathrm{S}$ & NSV & NSV & NSV & NSV \\
\hline \multicolumn{6}{|l|}{ Apgar score } \\
\hline $1 \mathrm{~min}$ & 8 & 9 & 8 & 7 & 9 \\
\hline $5 \min$ & 10 & 10 & 9 & 9 & 10 \\
\hline Weight at birth - g & $2200(25-50$ p) & $3100(50-75 p)$ & $2850(25-50 p)$ & $2100(75-90$ p) & $2400(25-50 p)$ \\
\hline Length at birth-cm & $46(25-50 p)$ & $49(50-75 p)$ & $48(25-50 p)$ & $43(50-75 p)$ & $48(25-50 p)$ \\
\hline Head circumference-cm & $32(50-75 p)$ & $34(50-75 p)$ & $33(25-50 p)$ & $32(75-90 p)$ & $33(25-50 p)$ \\
\hline System examination & Tachypnea & Normal & Normal & Tachypnea & Normal \\
\hline Symptom and signs & $\begin{array}{l}\text { irritability, high- } \\
\text { pitched cry, mild } \\
\text { tremors undis- } \\
\text { turbed, generalized } \\
\text { convulsions, fre- } \\
\text { quent yawning, } \\
\text { nasal flaring }\end{array}$ & $\begin{array}{l}\text { irritability hyperac- } \\
\text { tive Moro reflex, } \\
\text { mild tremors dis- } \\
\text { turbed, excessive } \\
\text { sucking, high- } \\
\text { pitched cry }\end{array}$ & $\begin{array}{l}\text { irritability, high- } \\
\text { pitched cry, mild } \\
\text { tremors undis- } \\
\text { turbed, myoclonic } \\
\text { jerks, frequent } \\
\text { yawning, nasal } \\
\text { flaring }\end{array}$ & $\begin{array}{l}\text { irritability, high- } \\
\text { pitched cry, mild } \\
\text { tremors undis- } \\
\text { turbed, generalized } \\
\text { convulsions, fre- } \\
\text { quent yawning, } \\
\text { nasal flaring }\end{array}$ & $\begin{array}{l}\text { irritability, high- } \\
\text { pitched cry, mild } \\
\text { tremors undis- } \\
\text { turbed, myoclonic } \\
\text { jerks, frequent } \\
\text { yawning }\end{array}$ \\
\hline Finnegan score & 15 & 5 & 11 & 15 & 10 \\
\hline Convulsions & + & - & - & + & - \\
\hline Medication & Phenobarbital & - & - & Phenobarbital & - \\
\hline Feeding & Formula & Breast feeding & Breast feeding & Formula & Breast feeding \\
\hline $\begin{array}{l}\text { Duration of infant's } \\
\text { hospital stay - days }\end{array}$ & 14 & 6 & 6 & 14 & 6 \\
\hline
\end{tabular}

\section{Case 1}

This baby boy was born vaginally as the fifth child of the family, at the $35^{\text {th }}$ week of gestation due to premature contractions. Apgar scores at the $1^{\text {st }}$ and $5^{\text {th }}$ minutes were 8 and 10 , respectively. The mother had used heroin daily before and during the pregnancy. Hepatitis $\mathrm{C}$ virus (HCV) serology of the mother was positive. The other children and members of the family were healthy. The physical examination revealed respiratory distress with intercostal retractions. The chest X-ray and laboratory findings were compatible with transient tachypnea of neonate (TTN). Nasal CPAP treatment was required. Due to the prolonged rupture of membranes and maternal urinary tract infection, intravenous ampicillin and gentamicin treatments were started. The initial laboratory tests were normal (complete blood count, glucose, sodium, potassium, calcium levels). On the second day of life (DOL), irritability, high-pitched cry, mild tremors undisturbed, generalized convulsions, frequent yawning, nasal flaring, respiratory rate $>60 /$ min with retractions were observed. The Finnegan neonatal abstinence score was 15 and phenobarbital treatment was started as a loading dose of $20 \mathrm{mg} / \mathrm{kg}$ and as a maintenance dose of $5 \mathrm{mg} / \mathrm{kg} / \mathrm{d}$. Complete blood count, routine blood biochemical tests, urine analysis, and thyroid function test re- sults were normal. The cranial ultrasonography and echocardiographic evaluation of the infant were normal. The withdrawal score fell to 10 in the follow-up, no convulsion was observed and withdrawal scores were less than 7 at discharge. As the mother was still abusing heroin, the baby was fed with formula. Phenobarbital treatment was stopped on the ninth DOL and the patient was discharged on the $14^{\text {th }}$ DOL.

\section{Case 2}

This baby girl was born by cesarean section as the second child of the family, at the $38^{\text {th }}$ week of gestation with Apgar scores of 9 and 10 at the 1 st and $5^{\text {th }}$ minutes, respectively. The mother was suffering from heroin addiction daily before and during the pregnancy. The other children and members of the family were healthy. The physical examination was normal. On the second DOL, irritability hyperactive Moro reflex, mild tremors disturbed, excessive sucking, high-pitched cry were observed. The Finnegan neonatal abstinence score was 5 and no treatment was started. The initial laboratory tests including complete blood count, glucose, sodium, potassium, calcium levels, cranial ultrasonography and echocardiographic evaluation were normal. The baby was fed with breast milk and was discharged on the $6^{\text {th }}$ DOL. 


\section{Case 3}

This baby boy was born vaginally as the third child of the family, at the $39^{\text {th }}$ week of gestation. Apgar scores at the 1 st and $5^{\text {th }}$ minutes were 8 and 9 , respectively. The mother was heroin addicted daily before and during the pregnancy. The other children and members of the family were healthy. The initial physical examination was normal. On the second DOL, irritability, high-pitched cry, mild tremors undisturbed, myoclonic jerks, frequent yawning, nasal flaring were observed. The initial laboratory tests including complete blood count, glucose, sodium, potassium, calcium levels, cranial ultrasonography and echocardiographic evaluation revealed normal results. The Finnegan neonatal abstinence score was 11 and no treatment was started. The baby was fed with breast milk and was discharged on the $6^{\text {th }}$ DOL.

\section{Case 4}

This baby girl was born vaginally as the first child of the family, at the $32^{\text {th }}$ week of gestation due to premature contractions. Apgar scores at the 1 st and $5^{\text {th }}$ minutes were 7 and 9 , respectively. The mother had used heroin daily before and during the pregnancy. The other children and members of the family were healthy. The physical examination of the infant revealed mild respiratory distress and oxygen treatment was required. Due to the prolonged rupture of membranes intravenous ampicillin and gentamicin treatments were started. On the second DOL, irritability, high-pitched cry, mild tremors undisturbed, generalized convulsions, frequent yawning, nasal flaring, respiratory rate $>60 / \mathrm{min}$ with retractions were observed. The Finnegan neonatal abstinence score was 15, and phenobarbital treatment was initiated as $20 \mathrm{mg} / \mathrm{kg}$ loading and $5 \mathrm{mg} / \mathrm{kg} / \mathrm{d}$ maintenance dose. The initial laboratory tests including complete blood count, glucose, sodium, potassium, calcium levels, cranial ultrasonography and echocardiographic evaluation were normal. The withdrawal score fell to 9 in the follow-up, no convulsion was observed and withdrawal score was less than 7 at discharge. As the mother was still abusing heroin and the baby was fed with formula. Phenobarbital treatment was stopped on the $9^{\text {th }}$ DOL, and the baby was discharged on the $14^{\text {th }}$ DOL.

\section{Case 5}

The patient was born vaginally as the third child of the family, at the $39^{\text {th }}$ week of gestation. Apgar scores at the 1 st and $5^{\text {th }}$ minutes were 9 and 10 , respectively. The mother was suffering from heroin addiction daily before and during the pregnancy. Although the initial physical examination was normal, on the second DOL, irritability, high-pitched cry, mild tremors undisturbed, myoclonic jerks, frequent yawning were observed. The Finnegan neonatal abstinence score was 10 and no treatment was started. The initial laboratory tests including complete blood count, glucose, sodium, potassium, calcium levels, cranial, abdominal ultrasonography and echocardiographic evaluation were normal. The baby was fed with breast milk and was discharged on the $6^{\text {th }}$ DOL.

\section{Discussion}

Maternal drug abuse has increased over the past decades. In the United States, substance use among pregnant and postpartum women is a public health issue. In 2011-2012, the National Survey on Drug Use and Health (NSDUH) in the United States found that $5.9 \%$ of pregnant women aged $15-44$ years reported using illicit drugs, compared with $10.7 \%$ of non-pregnant women in this age group (7). The rate of illicit drug use among pregnant women aged 15-17 years was $18.3 \%, 9 \%$ among pregnant women aged $18-25$ years, and $3.4 \%$ among pregnant women aged $26-44$ years (7). The most commonly used substances in pregnancy are cannabis, opiates and cocaine. Heroin used to be the most common opiate abused in pregnancy, but it is now methadone (3). Although there is no reported study about the incidence of drug abuse during pregnancy in our country, opioid abuse during pregnancy is increasing in women of childbearing age in Turkey. These five infants were treated in our institution only in oneyear period due to heroin abstinence syndrome. The frequency of NAS seems to be increased in future.

Most illicit drugs cause an addiction in the mother and dependence in the infant. Dependence or tolerance in the latter is due to passage of the drugs across the placental barrier; this occurs in varying degrees, depending on the pharmacokinetic properties of the individual drugs. Substances that act on the CNS are usually highly lipophilic and have relatively low molecular weight. These characteristics facilitate crossing from maternal to fetal circulation, with rapid equilibration of free drug between mother and fetus. Once drugs cross the placenta, they tend to accumulate in the fetus because of the immaturity of the renal function and the enzymes used for metabolism. Disruption of the transplacental passage of drugs at birth results in the development of a withdrawal syndrome (8).

The timing of onset of the symptoms gives an indication of the maternal drug abuse. Heroin has a short half-life and withdrawal also occurs within 48-72 $\mathrm{h}$ of birth, whereas methadone withdrawal occurs at 7-14 days. Neonatal abstinence syndrome is often a multisystem disorder that frequently involves the CNS, GI system, autonomic system, and respiratory system. Manifestations of NAS depend on various factors, including the drug used, its dose, frequency of use, and the infant's own metabolism and excretion of the active compound or compounds. Central nervous system and gastrointestinal system symptoms are the leading symptoms respectively (9). Convulsions, tremor, and hyperirritability are the most frequent findings. The infants are hyper-excitable, cannot sleep for a long time, and have a high-pitched cry. They have excessive sucking $(10,11)$. Central nervous system 
symptoms are followed by gastrointestinal symptoms. Feeding problems like abdominal distention and vomiting are common (11). Tachypnea and retractions, fever, frequent yawning, sneezing, nasal stuffiness, sweating, peeling of the skin, increased Moro reflex, and increased muscle tone are some of the other symptoms (11). Central nervous system symptoms were also the leading symptoms in our patients.

\section{Preterm infants have}

been described as being at lower risk of drug withdrawal with less severe and/or prolonged courses. Lower gestational age correlated with a lower risk of neonatal withdrawal. The apparent decreased severity of signs in preterm infants may relate to developmental immaturity of the CNS, differences in total drug exposure, or lower fat depots of drug. Alternatively, the clinical evaluation of the severity of abstinence may be more difficult in preterm infants, because scoring tools to describe withdrawal were largely developed in term or late preterm infants (8). Two of our cases were preterm and were evaluated by Finnegan abstinence scoring system.

Health care professionals, hospitals, and clinics have an obligation to assess newborns who exhibit signs and symptoms of drug exposure, whose mothers have been identified as probable substance users, or whose mothers have signs and symptoms of drug use. The diagnosis of prenatal opioid exposure is based upon a positive opioid use of the mother. Laboratory tests are less helpful in the diagnosis. Blood and urine tests can give false-positive or false-negative results. Meconium analysis is currently considered the best method for detecting drug exposure in pregnancy. It provides a wider window of detection of gestational exposure, presumably as remote as the second trimester, when drugs begin to accumulate in meconium, but implementation of the test is difficult. The baby's or the mother's hair analysis reflects the last trimester but is expensive and can be implemented in only a small number of centers $(3,4)$. In our cases, maternal drug abuse stories were apparent, so the diagnoses were performed easily based on the Finnegan scoring system, but we could not confirm the diagnosis with laboratory tests due to the lack of capability in our institution.

The average number of delivery per year in our hospital is 20000. Approximately 2000 babies are hospitalized. A prospective study should be performed to reveal the real incidence of the deliveries from maternal drug abuse.

Special attention should be paid to signs of intrauterine growth retardation (IUGR), microcephaly, prematurity, congenital infection, and major and minor congenital malformations (12). Two of the infants were preterm, none of the infants was small for gestational age. We did not detect any congenital anomaly neither in physical examination nor in radiological and echocardiographic evaluation.

Primary treatment of neonatal symptoms related to prena- tal substance exposure should be supportive because pharmacologic therapy can prolong hospitalization and exposes the infant to additional agents that are often not necessary. Pharmacotherapy for infants with more severe expression of NAS is necessary to allow them to feed, sleep, gain weight, and interact with caregivers. Approximately $30-91 \%$ of infants who exhibit signs of NAS receive pharmacological treatment. Medications used in patients with NAS should be considered when supportive measures like; minimizing environmental stimuli by reducing light exposure, minimizing excessive noise, avoiding unnecessary handling, swaddling, and non-nutritive sucking with a pacifier, frequent feeding with hypercaloric formula fail to ameliorate the infant's withdrawal. This may be manifested early on as difficulty with feeding, extreme irritability, and poor sleeping. If a scoring system is used, pharmacological treatment is commonly started when the average of 3 scores is 8 or more on the Finnegan scale (4). The optimal treatment for NAS has not been established. This is reflected in the considerable heterogeneity in the pharmacologic treatment of NAS among different institutions. Many pharmacological agents have been used to treat NAS. However, few randomized trials have compared the efficacy of the various pharmacological treatments. For opioid related NAS, morphine and methadone are given as substitutes. Non-morphine treatments (e.g., phenobarbital, chlorpromazine, diazepam, clonidine) provide symptomatic relief.

Opioids are currently considered the first-line therapy. The treatment for morphine administration has been reported to last 8-79 days (13). This length of hospitalization interferes with maternal bonding, has potential for nosocomial infection, and is a major use of resources (14). Second-line therapy has been phenobarbital. Phenobarbital has been effective for the treatment of opioid withdrawal seizures. Disadvantages include lack of effect on GI symptoms. We used phenobarbital in two cases who had convulsions and the phenobarbital treatment was effective. Sedative agents such as chloral hydrate, chlorpromazine, diazepam, and phenobarbitone can also be used in the treatment of withdrawal syndrome. Their effects are non-specific. Clonidine has been shown to be an effective and safe second-line medication for the treatment of NAS symptoms refractory to opioid therapy (15).

The American Academy of Pediatrics (AAP), The American College of Obstetricians and Gynecologists (ACOG), and the Academy of Breastfeeding Medicine (ABM) support breastfeeding among opioid-dependent women if the women are enrolled in substance abuse treatment and no contraindications to breastfeeding are observed, such as ongoing drug use or HIV infection $(16,17)$. Among our five infants two of them who were fed with formula presented with convulsion. As a speculation formula feeding may cause a rapid decrease in the heroin level of the infants. So, we thought that even in ongoing drug use breastfeeding may slow down the decrease of opioid level in blood and may reduce the symptoms. 


\section{Conclusion}

Opioid abuse in pregnancy and NAS are growing sociological problems in Turkey. We would like to alert the clinicians that in the presence of central nervous system and gastrointestinal system symptoms of unknown origin, NAS should be kept in mind. A practical guideline for diagnosis and management of NAS is required for neonatologists in Turkey. For the institutions which could not perform evidence based diagnosis, treatment and follow up we recommend breastfeeding to slow down the decrease of opioid level in blood and to reduce the symptoms.

: Acknowledgements: None

Financial / Material Support: None

Conflict of Interest: The authors declare no conflict of interest.

\section{References}

1. Alpan G. Infant of a drug abusing mother. In: Gomella TL, Cunningham MD, Eyal FG, Zenk KE, editors. Neonatology: Management, Procedures, On-Call Problems, Diseases, and Drugs. New York: McGraw Hill Medical 2009;540-9.

2. Kale-Çekinmez E, Mutlu B, Yapıcıoğlu H, Özlü F, Asker $\mathrm{H}$, Mert K, et al. Two newborns of heroin-addicted mothers suffering neonatal withdrawal syndrome. Turk J Pediatr 2012;54(4):421-4.

3. Jansson LM, Velez M, Harrow C. The opioid exposed newborn: assessment and pharmacologic management. J Opioid Manag 2009;5(1):47-55

4. Finnegan LP. Neonatal abstinence syndrome: assessment and pharmacotherapy. In: Rubatelli. FF and Granati. B. Editors. Neonatal therapy: An update. New York, NY: Excerpta Medica 1986;122-46.

5. Ostrea EM, Ostrea AR, Simpson PM. Mortality within the first 2 years in infants exposed to cocaine, opiate, or cannabinoid during gestation. Pediatrics 1997;100(1):7983.

6. Lester BM, Tronick EZ. History and description of the Neonatal Intensive Care Unit Network Neurobehavioral Scale. Pediatrics 2004;113(3 Pt 2):634-40.

7. Substance Abuse and Mental Health Services
Administration. Results from the 2012 National Survey on Drug Use and Health: Summary of National Findings. US Department of Health and Human Services; September 2013. http://store.samhsa.gov/home.

8. Patrick SW, Schumacher RE, Benneyworth BD, Krans EE, McAllister JM, Davis MM. Neonatal abstinence syndrome and associated health care expenditures: United States, 2000-2009. JAMA 2012;307(18):1934-40.

9. Hudak ML, Tan RC. Neonatal Drug Withdrawal. Pediatrics 2012;129(2):e540-60.

10. Hulse GK, Milne E, English DR, Holman CD. Assessing the relationship between maternal opiate use and neonatal mortality. Addiction 1998;93(7):1033-42

11. Behnke M, Eyler FD. The consequences of prenatal substance use for the developing fetus, newborn and young child. Int J Addict 1993;28(13):1341-91.

12. Sherwood RA, Keating J, Kavvadia V, Greenough A, Peters TJ. Substance misuse in early pregnancy and relationship to fetal outcome. Eur J Pediatr 1999;158(6):48892.

13. Lainwala S, Brown ER, Weinschenk NP, Blackwell MT, Hagadorn JI. A retrospective study of length of hospital stay in infants treated for neonatal abstinence syndrome with methadone versus oral morphine preparations. Adv Neonatal Care 2005;5(5):265-72.

14. Kraft WK, Gibson E, Dysart K, Damle VS, Larusso JL, Greenspan JS, et al. Sublingual buprenorphine for treatment of neonatal abstinence syndrome: a randomized trial. Pediatrics 2008;122(3):e601-7.

15. Grim K, Harrison TE, Wilder RT. Management of neonatal abstinence syndrome from opioids. Clin Perinatol 2013;40(3):509-24.

16. Committee on Health Care for Underserved Women and the American Society of Addiction Medicine. ACOG Committee Opinion No. 524: Opioid abuse, dependence, and addiction in pregnancy. Obstet Gynecol 2012;119(5): 1070-6.

17. Academy of Breastfeeding Medicine Protocol Committee, Jansson LM. ABM clinical protocol \#21: Guidelines for breastfeeding and the drug-dependent woman. Breastfeed Med 2009;4(4):225-8. 